East African Medical Journal Vol. 83 No. 11 November 2006

SEASONAL VARIATION AND PREVALENCE OF TUBERCULOSIS AMONG HEALTH SEEKERS IN THE SOUTH WESTERN CAMEROON

I.N. Ane-Anyangwe, MSc, Lecturer, Department of Biochemistry and Microbiology, Faculty of Science, T.N. Akenji, $\mathrm{PhD}$, Associate Professor, Department of Medical Laboratory Science, Faculty of Health Sciences, University of Buea, P.O. Box 63, Buea, Southwest Province, Cameroon, W.F. Mbacham, ScD, Associate Professor, V.N. Penlap, PhD, Senior Lecturer, Biotechnology Centre, University of Yaoundé 1, Yaoundé, Cameroon and V.P.K.Titanji, PhD, C Biol., FI Biol, Professor, Department of Biochemistry and Microbiology, Faculty of Science, University of Buea, P.O. Box 63, Buea, Southwest Province, Cameroon

Request for reprints to: Professor V.P.K.Titanji, Department of Biochemistry and Microbiology, Faculty of Science, University of Buea, P.O. Box 63, Buea, South West Province, Cameroon

\title{
SEASONAL VARIATION AND PREVALENCE OF TUBERCULOSIS AMONG HEALTH SEEKERS IN THE SOUTH WESTERN CAMEROON
}

\author{
I.N. ANE-ANYANGWE, T.N. AKENJI, W.F. MBACHAM, V.N. PENLAP and V.P.K.TITANJI
}

\begin{abstract}
Objectives: To determine the prevalence of tuberculosis (TB) in Fako health District, to assess the effects of seasonal variation on the incidence of $\mathrm{TB}$ in the study area and to use sentinel analysis to predict areas of greatest infection.

Design: A prospective cross sectional study based on laboratory investigations.

Setting: Fako health District, South Western Carneroon.

Results: The prevalence of TB was $23.3 \%$.Tuberculosis was significantly more prevalent in males $(12.6 \%)$ as compared with females $(10.7 \%)(P=0.034)$. TB prevalence was significantly different between age groups, with the highest number of cases recorded in the age group 21-30 ( $P=0.002)$. When the health areas were compared, TB prevalence varied significantly $(P=0.001)$, with Limbe Town recording the highest number of TB cases. We recorded more TB cases in the wet season compared with the dry season and the difference was statistically significant $(P=0.000)$. There was a significant drop in the prevalence of TB over the study period $(P=0.000)$.

Conclusion: This study is the first to report on the effects of season on the prevalence of TB in Cameroon. These findings will therefore provide additional useful base line data for setting up TB control strategies in Cameroon.
\end{abstract}

\section{INTRODUCTION}

Tuberculosis (TB) is increasingly becoming a world wide problem because of the advent of HIV and AIDS, poor living conditions (1) and the emergence of multi-drug-resistance (MDR) TB for which treatment is beyond the reach of most African countries. One third of the world's population is exposed to $\mathrm{TB}$ and it is responsible for approximately three million deaths each year. It is estimated that eight million new cases of TB occur each year and Africa has the highest incidence rate (2). In Cameroon, a country with 15 million inhabitants, the prevalence of TB is estimated to be $17.8 \%$ and the incidence in 2000 was estimated to be over 300 cases per 100,000 inhabitants with an estimated 21,594 new sputum smear-positive cases (2).

Some work has been done in Cameroon especially on drug resistance to $\mathrm{TB}(3,4)$, morbidityassociated factors (5), TB/HIV as co-morbid infection (4), rapid diagnosis using PCR, molecular typing and genetic diversity (6). To date, no studies have been performed to determine the effects of seasons on prevalence of TB in South Western Cameroon, a major agro industrial zone of the 
country. Most people in this area live in agro industrial camps, which are generally over crowded and therefore is a predisposing factor for TB in the area. Seasonal fluctuation of TB has been reported in some countries (7-10). Data on the seasonality of TB is available in most developing countries including Cameroon is inadequate. This is worsened by the lack of resources for diagnosis and treatment of TB. Hippocrates once said "Whoever wishes to investigate medicine properly should proceed thus: in the first place to consider the seasons of the year, and what effects each of them produces" (10). With this in mind, the aim of the study was to determine the prevalence of TB in the Fako Health District of the South West Province of Cameroon and to find out the effects of seasonal changes on the transmission of the disease. We also sought to use sentinel analysis to predict areas of greatest infection.

\section{MATERIALS AND METHODS}

Study site and period: The study was performed over a 27 month period (April 2002 to July 2004) in three major hospitals (the Limbe District Hospital, Buea General Hospital. and Tiko Cottage Hospital) in Fako Division of the South West Province of Cameroon. Fako Division has a population of about 330,692 inhabitants and was chosen because the national TB control programme was first set up in this area in 2002. This Health District was sub grouped into the following Health areas as determined by the Delegation of Public Health, South West Province Cameroon: Bojongo, Bokwango, Bonadikombo, Bota, Bova, Buea Town, ldenau, Likomba, Limbe Town, Limbe Sea Port, Missellele, Molyko, Mondoni, Muea, Mutengene, Muyuka, Soppo, Tiko Town and Tole.

Demographic data: Demographic data (Age, sex, residence and ethnic origin) were collected from all 2,809 patients who were referred to the TB laboratories in these hospitals using a structured questionnaire.

Admission criteria and informed consent: After the patient's or guardian's consent, early morning sputum samples $(0.5-1 \mathrm{ml})$ were collected from the patients in the TB laboratories of the various hospitals. Ethical clearance for this work was obtained from the South West Provincial Delegation of Public Health.

Seasons and rainfall: In South West Cameroon, the rainy season lasts from April to November and the dry season, from November to March. Records of the mean monthly rainfall during the study period (April 2002 to July 2004) were obtained from the agro-industrial company, namely, the Cameroon Development Cooperation (CDC) in Tole.

Clinical samples: A total of 2,809 sputum samples were collected into sterile screw capped tubes from all patients reported with one or several of the following symptoms: persistent cough for over three weeks; night sweats and fever; loss of $10 \%$ or more of body weight and HIV infection. Smears were prepared and the slides were stained with ZiehlNeelsen stain (11) and viewed under a light microscopy at X1000 magnification.

Statistical analysis: The data were analysed using Microsoft Excel 2003 and SPSS for Windows version 13.0. The Pearson's Chi square test was used to compare qualitative variables. Statistical significance was set at $P \leq 0.05$.

\section{RESULTS}

The prevalence of tuberculosis in the Fako Health District: Of the 2,809 subjects examined in Limbe, Tiko and Buea, 1,416 were males and 1,393 females. The overall prevalence of TB and the prevalence by health area are shown on Table 1 . The prevalence of TB differed significantly between sexes and was $12.6 \%$ for males and $10.7 \%$ for females $\left(\mathrm{x}^{2}=4.5, \mathrm{P}=0.034\right)$.

Limbe Town recorded the highest number of cases $(193 / 719,24.4 \%)$, followed by Tiko Town, (84/ $539,15.6 \%)$, Muyuka (68/228, 29.8\%), Buea Town (51/240, 21.25\%), Mutengene (45/169, 26.6\%) and ldenau $(43 / 164,26.2 \%)$. Lower number of cases came from Bonadikombo (2/13, 15.4\%), Missellele $(4 / 28,14.3 \%)$, Bokwangwo (5/31, 16.1\%), Mondoni $(6 / 34,17.6 \%)$ and Likomba $(6 / 20,30.0 \%)$. There was a significant difference in the prevalence of TB between Health areas $\left(x^{2}=44.2, P=0.000\right)$.

Sentinel study: The number of TB cases within quarters in the Health areas is indicated in Figure 1. 
Counts from 1-33 were considered low, 34-65 moderate and $>65$ high. In Limbe, quarters like New Town, Unity quarter and C.D.C. Bota recorded higher number of TB cases. In Tiko, high counts were recorded in C.D.C camp and Mutengene while in Buea, Buea Town registered the highest number of TB cases (Figure 1). Village settings like Mondoni, Bonadikombo with subsistence farming as the main activity and relatively affluent neighbourhoods like Bokwangwo and Bonduma registered a low prevalence (Figure 1, Table 1).

Effect of age on the prevalence of TB: TB prevalence was statistically different among age groups. The age stratified prevalence curve was bell shaped
(Figure 2), with TB prevalence rising slowly in the first and second decades to about $35.5 \%$ in the third decade, followed by $29.5 \%$ in the fourth decade. Thereafter there was a dramatic decline.

Seasonal changes in TB prevalence: The number of TB cases varied significantly between seasons over the years with more cases recorded in the rainy season (163 positive cases in 2002; 172 in 2003 and 80 in 2004) compared with the dry season (47 positive cases in 2002; 132 in 2003 and 58 in 2004) and the difference was statistically significant $\left(x^{2}=23.8\right.$, $\mathrm{P}=0.000$ ) (Figure 3).

TB prevalence followed rainfall trend and peaked with mean monthly rainfall (Figures $4 \mathrm{a}, \mathrm{b}, \mathrm{c}$ ).

Table 1

Prevalence of tuberculosis among health care seekers in the Fako Health District

\begin{tabular}{|c|c|c|c|c|c|c|c|}
\hline \multirow[t]{2}{*}{ Study area } & \multicolumn{3}{|c|}{ Subjects enrolled } & \multicolumn{3}{|c|}{ TB positives } & \multirow{2}{*}{$\begin{array}{c}\text { Prevalence } \\
(\%)\end{array}$} \\
\hline & Male & Female & Total & Male & Female & Total & \\
\hline Bojongo & 23 & 28 & 51 & 9 & 8 & 17 & 33.3 \\
\hline Bokwoango & 15 & 16 & 31 & 2 & 3 & 5 & 16.1 \\
\hline Bonadikombo & 8 & 5 & 13 & 1 & 1 & 2 & 15.4 \\
\hline Bota & 49 & 45 & 94 & 13 & 12 & 25 & 26.6 \\
\hline Bova & 12 & 17 & 29 & 4 & 5 & 9 & 31.0 \\
\hline BueaTown & 117 & 123 & 240 & 30 & 21 & 51 & 21.3 \\
\hline ldenau & 88 & 76 & 164 & 25 & 18 & 43 & 26.2 \\
\hline Likomba & 14 & 6 & 20 & 4 & 2 & 6 & 30.0 \\
\hline Limbe Town & 351 & 368 & 719 & 105 & 88 & 193 & 26.8 \\
\hline Limbe Sea Port & 44 & 32 & 76 & 14 & 7 & 21 & 27.6 \\
\hline Missellele & 18 & 10 & 28 & 3 & 1 & 4 & 14.3 \\
\hline Molyko & 29 & 43 & 72 & 6 & 9 & 15 & 20.8 \\
\hline Mondoni & 14 & 20 & 34 & 2 & 4 & 6 & 17.6 \\
\hline Muea & 59 & 98 & 157 & 19 & 18 & 37 & 23.6 \\
\hline Mutengene & 74 & 95 & 169 & 19 & 26 & 45 & 26.6 \\
\hline Muyuka & 102 & 126 & 228 & 31 & 37 & 68 & 29.8 \\
\hline Soppo & 41 & 36 & 77 & 7 & 4 & 11 & 14.3 \\
\hline Tiko Town & 317 & 222 & 539 & 49 & 35 & 84 & 15.6 \\
\hline Tole & 41 & 27 & 68 & II & 2 & 13 & 19.1 \\
\hline Total & 1416 & 1393 & 2809 & 354 & 301 & 665 & 23.3 \\
\hline
\end{tabular}




\section{Figure 1}

Map of Fako Health District showing the number of TB by age group

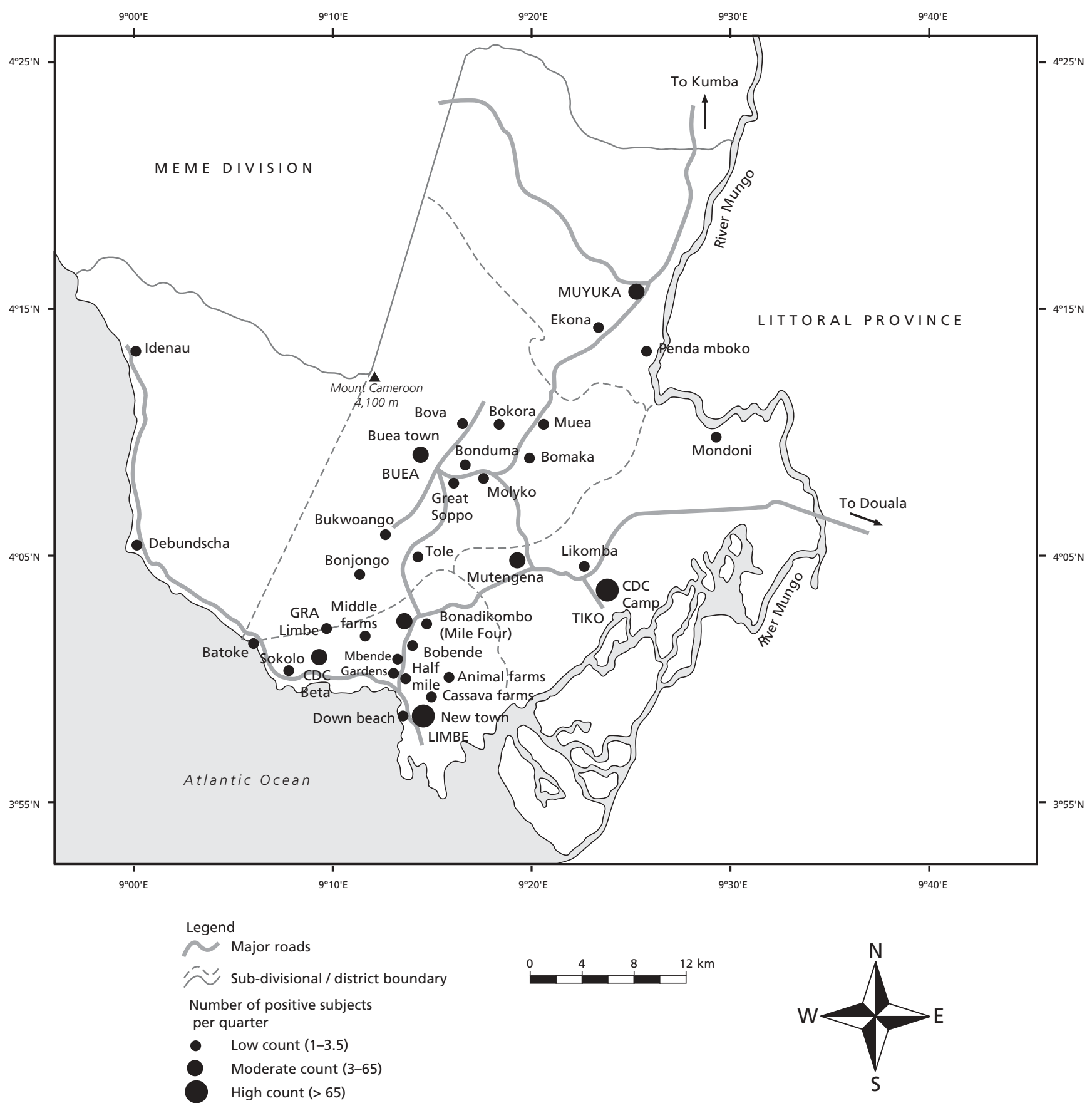

Map of Fako Health District showing the number of TB positive subjects within quarters in the health areas. Counts from 1-33 were considered low, 34-65 moderate and $>65$ high 
Figure 2

Prevalence of TB by age group

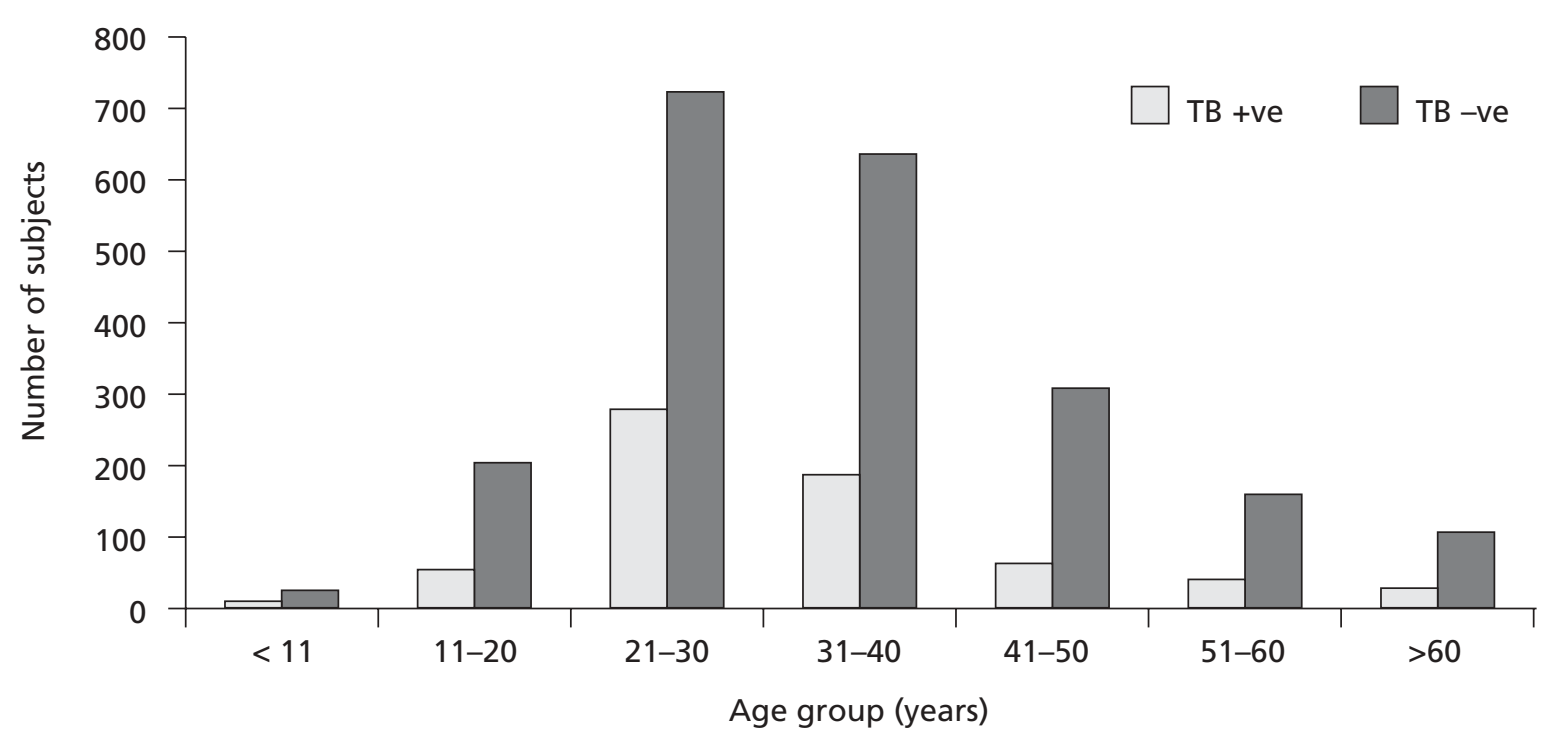

This shows the age stratified prevalence of TB. The curve showed a doom bell shape with TB prevalence rising slowly in the first and second decades to about $35.5 \%$ in the third decade, followed by $29.5 \%$ in the fourth decade. Thereafter there was a dramatic decline.

Figure 3

Cases of TB between the dry season (DS) and rainy season (RS) during the study period in the different years

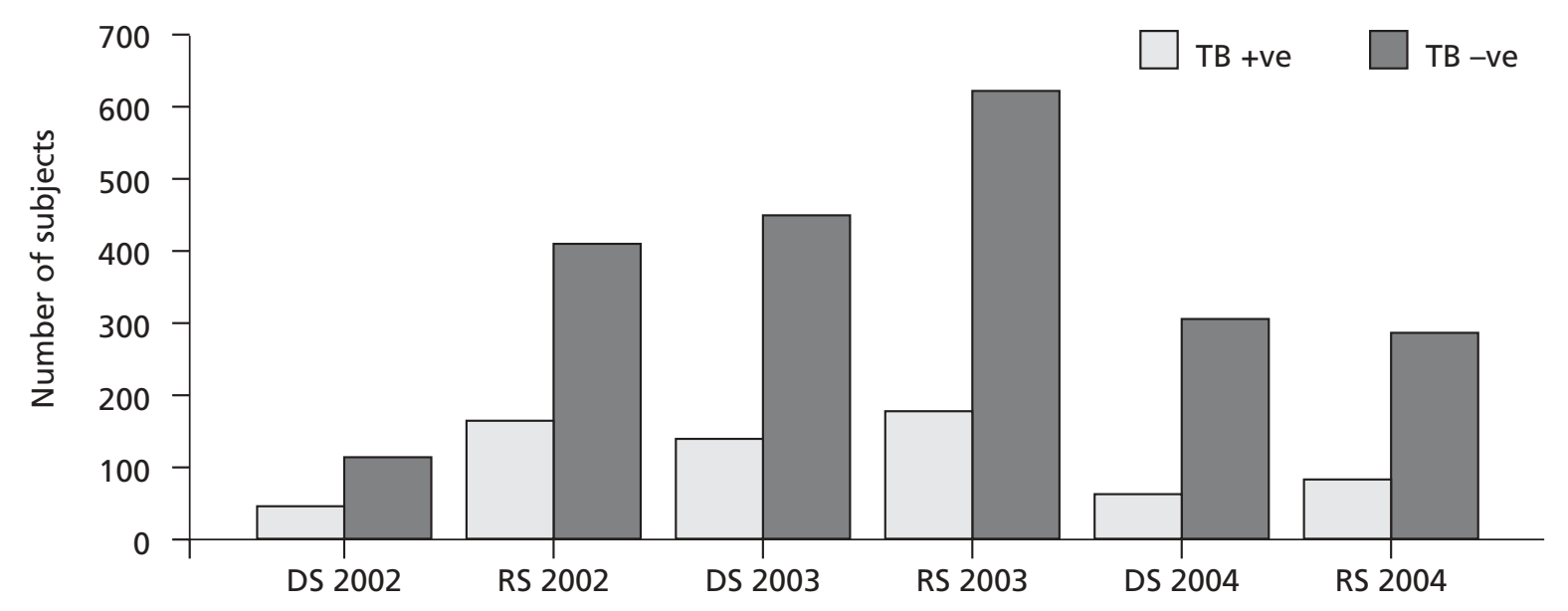

The graph shows the number of TB cases over the years. More cases were recorded in the rainy season (163 positive cases in 2002; 172 in 2003 and 80 in 2004) compared with the dry season (47 positive cases in 2002; 132 in 2003 and 58 in 2004) and the difference was statistically significant ( $P=0.000)$. 


\section{Figure 4a}

Prevalence of TB and mean monthly rainfall in 2002

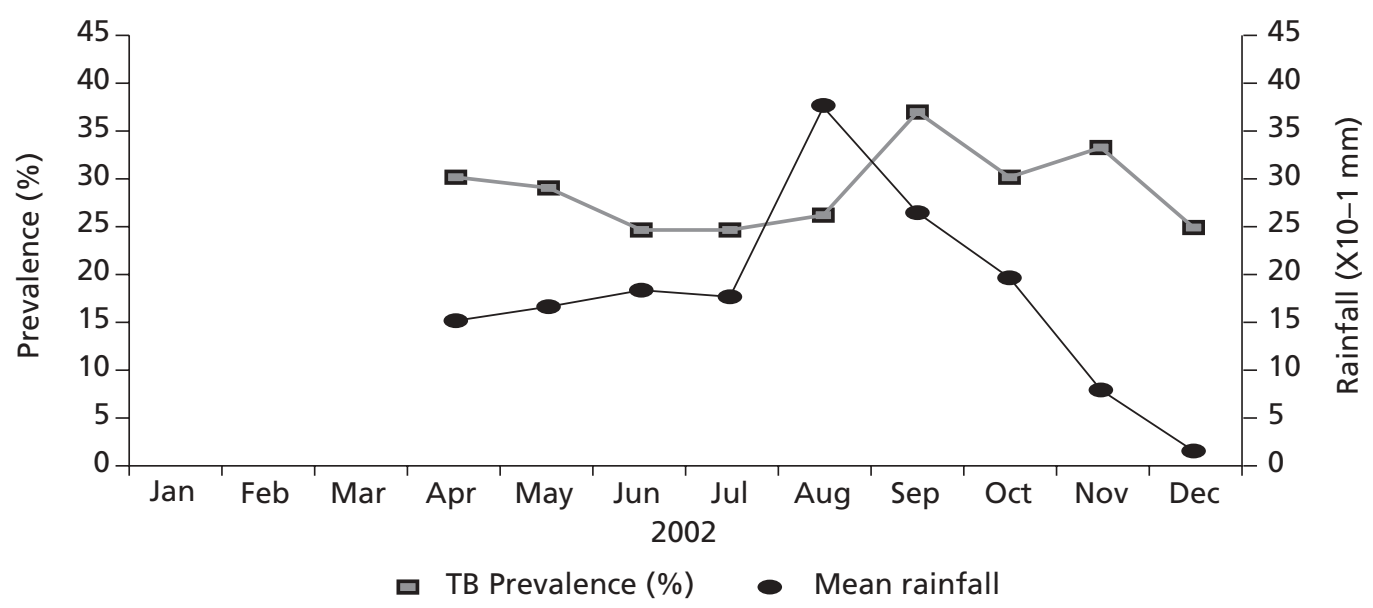

Figure $4 b$

Prevalence of TB and mean monthly rainfall in 2003

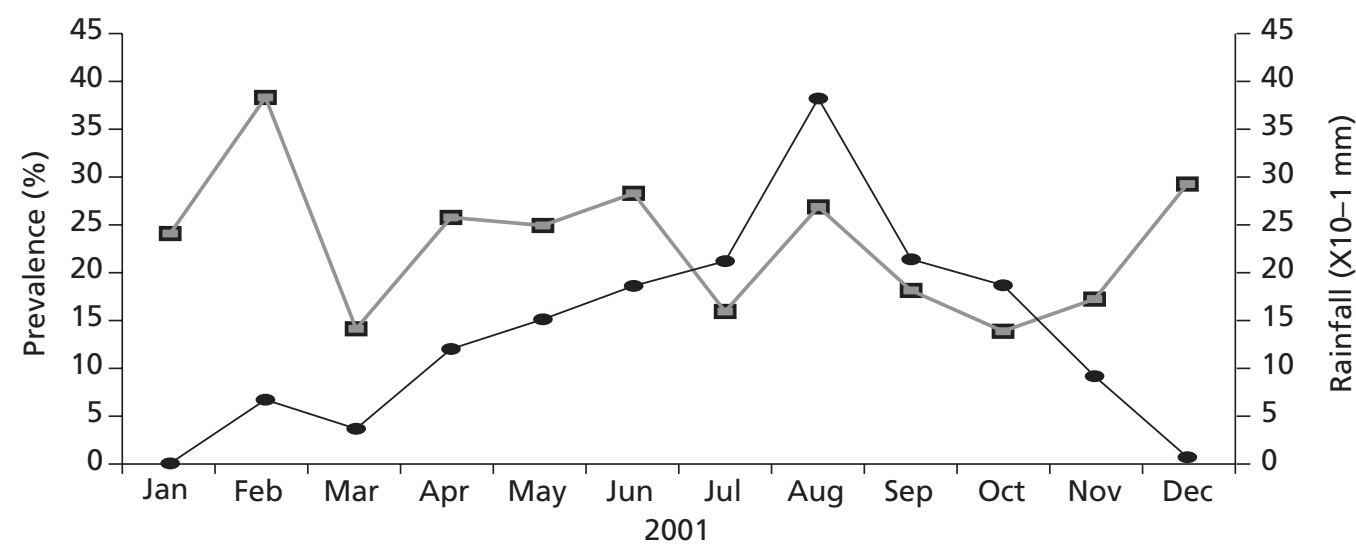

口 TB Prevalence (\%) @ Mean rainfall

Figure $4 \mathrm{c}$

Prevalence of TB and mean monthly rainfall in 2004

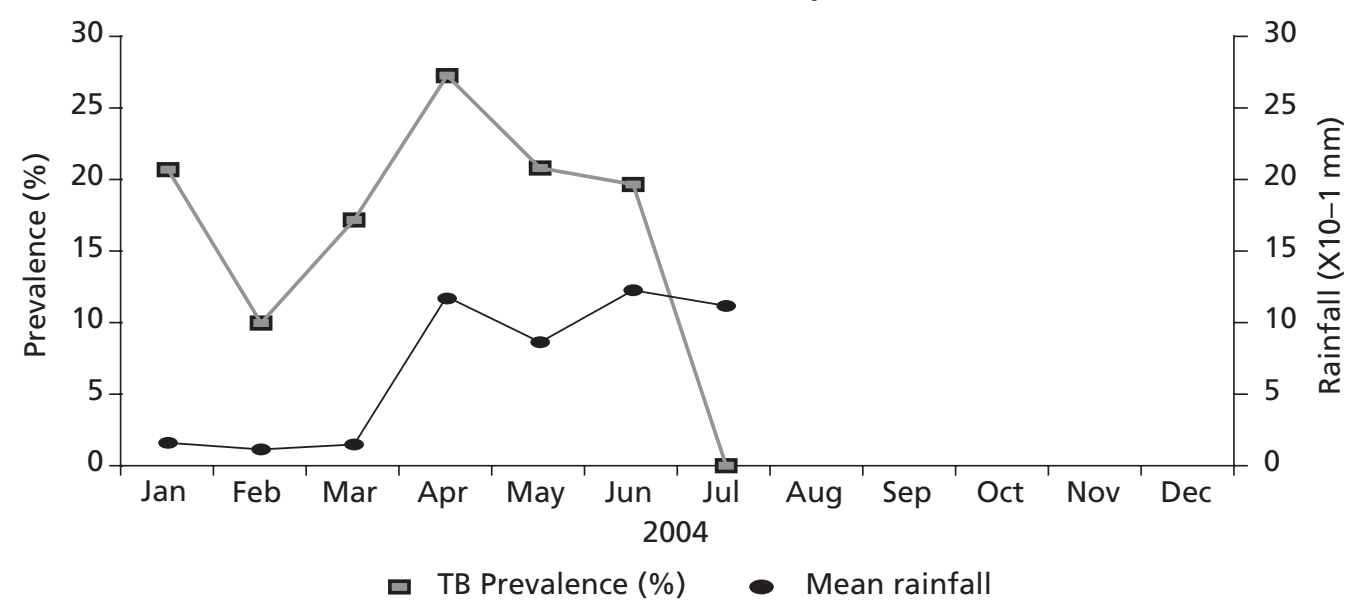

Figures $4 \mathrm{a}, \mathrm{b}$ and $\mathrm{c}$ show that the prevalence TB followed rainfall trend and peaked with mean monthly rainfall throughout the study period. 


\section{DISCUSSION}

Our study aimed at finding out the effect of seasonal changes in the epidemiology of the disease and to identify the localities in our study area which show high prevalence. The significantly high prevalence of TB in the rainy season compared with the dry season as recorded in this study corroborates the findings in other countries (7-10). Although we did not do a household survey to trace contact with relatives, close contact prevails mostly during the wet and cold period which keeps most people indoors and thus increases contact with infected relatives. It has also been reported that vitamin $\mathrm{D}$ deficiency predisposes subjects to TB. Vitamin D is important in the host's defence against TB. Lack of sunlight, which is common in the rainy season, may compromise the level of vitamin D in the body and thus lead to a weaker immune system against $\mathrm{TB}$ $(12,13)$. However; levels of vitamin D were not monitored in our subjects.

This study clearly shows that TB is still an important health problem in the study area despite recent government sponsored control programmes.Tiko and Limbe Towns (Table 1) stood out as the most highly infected areas with high prevalences in various quarters within these localities. In the Limbe Health District, quarters like New Town, Unity quarter and C.D.C. Bota recorded the highest prevalence of TB, while in Tiko, C.D.C camp and Mutengene recorded high prevalences.

In Buea, Buea Town registered the highest prevalence of TB (Figure 1). The high prevalence in New Town-Limbe and C.D.C. Tiko (where plantation workers live) could be due to the presence of city markets, over-crowding (in plantation camps), low wages by plantation workers and HIV / AIDS (1) among other factors. ldenau is a local sea port area where boats transport people to neighbouring countries like Nigeria, Equatorial Guinea and Gabon. High prevalence of TB has been reported among immigrant populations (14). It is possible that due to commercial activities in this area, TB is imported from other areas. The relatively low prevalence in village settings like Mondoni, Bonadikombo and in relatively affluent neighbourhoods like Bokwangwo and Bonduma can be attributed to the spacious environments and therefore less overcrowding, which is a predisposing factor for TB.
The significantly high prevalence of TB in males recorded in this study when compared with females is not an unusual finding. Globally, more males than females have been reported to be TB positive (2). This has been attributed to their often more risky activities, which include high social interactions, love for overcrowded environments, drinking, and smoking which enhance the spread of TB (5). Despite the observed trend, it is hypothesized that with the spread of HIV, which disproportionately affects women, the proportion of female TB cases is likely to increase in highly affected areas (15).

In this study, a high prevalence of $23.3 \%$ was recorded among health seekers in Fako Division. Treatment is beyond reach of most Cameroonians and the Directly Observatory Treatment Scheme (DOTS) does not provide complete coverage of the territory. However, the proportion of TB cases decreased steadily over the study period. According to reports from the Provincial Delegation of Public Health in the South West Province of Cameroon, the DOTS went into full effect in this area in 2002. This partially explains the decline in the numbers of cases during the study period.

Despite efforts made to curb the spread of TB in most countries, TB is still known to be most prevalent in the elderly. This has been attributed to the reactivation of the latent infection acquired many years earlier (16). In the present study more TB cases were recorded in the age group 21-30 years, closely followed by the age group 31-40 years (Figure 2). This shift in trend to young adults and the middle aged as also reported in other studies (16) has been ascribed to the advent of HI V/AIDS, which is most prevalent in people in these age brackets (17). In Cameroon TB is the most common opportunistic infection associated with AIDS (4) which may account for the high prevalence in the middle age group. The BCG vaccination of newborn babies has been widely practised in Cameroon and offers protection against the disease (18). This could explain why individuals less eleven years had the lowest prevalence of TB.

The data from the study indicates strongly that season influenced the epidemiological pattern of TB. Furthermore, the present epidemiologic pattern of TB in Fako Division, strongly suggests that additional effort is needed to intensify the DOTS scheme in the various health care settings and localities. Because of the severity and consequences 
of the disease to public health, local control programmes should be instituted and major educational efforts made to improve public awareness of the disease and the impact of HIV/ AIDS on the spread of the disease. It would be important to consider the impact of seasonal changes in such evaluation strategies. Being aware that the rainy season is a period of high transmission, the community can be sensitised to observe and report any signs or symptoms of TB in any close person during this period for diagnosis and eventual treatment. This would be helpful as a control strategy.

\section{ACKNOWLEDGEMENTS}

To the University of Buea (Staff Development Grant No 2374/UB/ACA/DTTS/TSS) and the International Programme in the Chemical Science (IPICS/CAM 01) for providing the funds for this study.

\section{REFERENCES}

1. Kamuolratanakul P., Sawwert H. and Kongsin S. Economic impact of tuberculosis at the household level. Int. J. Tubercule Lung Dis. 1999; 3: 596-602.

2. World Health Organization. Global Tuberculosis Control: Surveillance, Planning and Financing. WHO/CDS/ TB/2002. No. 295. World Health Organization, Geneva, Switzerland.

3. Bercion R. and Kuaban C. Initial resistance to antituberculosis drugs in Yaounde, Cameroon. Int. J. Tubercle Lung Dis. 1997; 1: 110-114.

4. Kuaban C., Ndoumou A., Koualla-shiros S., Afaneze E., Ghipponi P. M. and Pignon D. Seroprevalence of HIV infection among patients with pulmonary tuberculosis in Yaounde-Cameroon. West Afr. J. Med. 1995; 14: 112-115.

5. Holtedahlk H.H. Cross sectional study of morbidity, morbidity-associated factors and cost of treatment in Ngoundere, Cameroon, with implications for health policy in developing countries and development assistance policy. Int. Hlth $\mathcal{E}$ Human Rights. 2002; 2: 2.
6. Penlap V., Titanji V., Mbacham W. and Tedom B.R. Rapid diagnosis of tuberculosis using PCR in Yaounde Cameroon. Cameroon J. Biol. Biochem. Sci. 1997; 7: 63-68.

7. Leung C.C., Yew W.W., Chan C.T., et al. Seasonal pattern of tuberculosis in Hong Kong. Int. J. Epidemiol. 2005; 34: 924-930.

8. RAos M.Â., GarcAa J.M.A., SAjnchez J.A.Â. and PAcrez D.Â. Statistical analysis of the seasonality in pulmonary tuberculosis. Eur. J. Epidemiol. 2000; 16: 483-488.

9. Schaaf H.S., Nel E.D., Beyers N., Gie R.P., Scott F. and Donald P.R. A decade of experience with mycobacterium tuberculosis culture from children: A Seasonal influence on incidence of childhood tuberculosis. Tubercle and Lung Dis. 1996; 77: 43-46.

10. Michael Bell., Lennox K.A., Nwanyanwu O., et al. Seasonal variation in the etiology of blood stream infection in a febrile inpatient population in developing country. Int.l J. Inf. Dis. 2001; 5: 63-69.

11. Narvaid de Kantor I., Kim S.J., Frieden T., et al. Laboratory Services in Tuberculosis Control Part 11. Microscopy. WHO/TB/89.258, Jatto Associati s.a.s.Biella, Italy. 1998.

12. Ustianowski A., Shaffer R., Collin S., Wilkinson R.J. and Davidson R.N. Prevalence and association of vitamin D deficiency in foreign-born persons with tuberculosis in London. J. Inf. 2004; 50: 432-437.

13. Douglas A.S., Ali S. and Bakhshi S.S. Does vitamin D deficiency account for ethnic differences in tuberculosis seasonality in UK? Ethnic Hlth. 1998; 3: 247-253.

14. UIf D., Sandven P., Einar H. and Dominique A.C. Molecular epidemiology of Mycobacterium tuberculosis. Norway J. Clin. Microbiol. 2001; 39: 1802-1807.

15. UNAIDS Facts-sheet. Kenya. UNAIDS. 14.

16. Thomas R., Frieden M.P.H., Paula I., et al. Tuberculosis in New York City -Turning the Tides. Special Article. 1995; 333: 229-233.

17. WHO Epidemiology. Tuberculosis and HIV. What is the evidence for an association between TB and HIV. Some questions and answers. 2002. p2.

18. Donald P.R. Children and Tuberculosis: Protecting the next generation. Lancet. 1999; 353: 1001-1002. 